\title{
Second order parabolic equations and weak uniqueness of diffusions with discontinuous coefficients
}

\author{
DOYOON KIM
}

\begin{abstract}
We prove the unique solvability of parabolic equations with discontinuous leading coefficients in $W_{p}^{1,2}\left((0, T) \times \mathbb{R}^{d}\right)$. Using this result, we establish the uniqueness of diffusion processes with time-dependent discontinuous coefficients.
\end{abstract}

Mathematics Subject Classification (2000): 35K10 (primary); 35R05, 60G44, $60 \mathrm{H} 10$ (secondary).

\section{Introduction}

The unique solvability of second order parabolic equations in non-divergence form is well established if the leading coefficients are uniformly continuous (see [8] and [9]). More precisely, the parabolic equation

$$
u_{t}=a_{j k}(t, x) u_{x_{j} x_{k}}+b_{j}(t, x) u_{x_{j}}+c(t, x) u+f(t, x)
$$

with appropriate initial and boundary conditions has a unique solution $u \in$ $W_{p}^{1,2}((0, T) \times \Omega), 1<p<\infty$, if $a_{j k}(t, x)$ are uniformly continuous with respect to $x$ uniformly in $t$ ( $a_{j k}$ satisfy the uniform ellipticity condition). However, even if $a_{j k}(t, x)$ are discontinuous, there are some cases where one can expect the unique solvability of the above equation. The most intensively investigated case is when the coefficients $a_{j k}$ are in the space of VMO. As noted in [13] (also see references therein to find a list of many papers which deal with elliptic and parabolic equations with VMO coefficients), VMO contains the space of bounded uniformly continuous functions as a proper subset. However, for example, if $a_{j k}$ are piecewise constant, then they are not in the space of VMO. This means that we may try to find another class of discontinuous coefficients $a_{j k}$ (a class that includes piecewise constant coefficients) so that equations with leading coefficients from the class have strong unique solutions.

Received June 20, 2005; accepted in revised form January 18, 2006. 
In this paper we prove the existence and uniqueness of a solution $u$ in $W_{p}^{1,2}((0, T) \times$ $\left.\mathbb{R}^{d}\right), 1<p<\infty$, to the above parabolic equation with coefficients $a_{j k}(t, x)$ being discontinuous at the hyper-plane $\left\{\left(t, 0, x^{\prime}\right): t \in \mathbb{R}, x^{\prime} \in \mathbb{R}^{d-1}\right\}$. Specifically, we assume that

$$
a_{j k}(t, x)=a_{j k}^{+}(t, x) \text { for } x_{1}>0 \text { and } a_{j k}(t, x)=a_{j k}^{-}(t, x) \text { for } x_{1}<0,
$$

where $a_{j k}^{+}(t, x)$ and $a_{j k}^{-}(t, x)$ are defined on $\overline{\mathbb{R} \times \mathbb{R}_{+}^{d}}$ and $\overline{\mathbb{R} \times \mathbb{R}_{-}^{d}}$ respectively and uniformly continuous with respect to spatial variables uniformly in $t$. In addition, $a_{j k}^{ \pm}\left(t, 0, x^{\prime}\right)$ are uniformly continuous as functions of $\left(t, x^{\prime}\right)$. We see that $a_{j k}^{+}$do not have any relation with $a_{j k}^{-}$, so the coefficients $a_{j k}$ can be discontinuous at the hyperplane $\left\{\left(t, 0, x^{\prime}\right): t \in \mathbb{R}, x^{\prime} \in \mathbb{R}^{d-1}\right\}$. Clearly, this contains piecewise constant $a_{j k}$ with discontinuity at the hyper-plane. An a priori estimate, as usual, is the main step we achieve, and to do that, we make use of trace operators and multipliers.

When $a_{j k}, b_{j}$, and $c$ are piecewise constant with discontinuity at the above hyper-plane, Salsa [12] solved in $W_{2}^{1,2}\left((0, \infty) \times \mathbb{R}^{d}\right)$ the equation $u_{t}=a_{j k} u_{x_{j} x_{k}}+$ $b_{j} u_{x_{j}}+c u$ with a non-zero initial condition. For elliptic equations with similar leading coefficients, see [10], [11], and [5].

In this paper we also discuss the well-posedness of the martingale problem for $L_{t}$, where

$$
L_{t}=\frac{1}{2} a_{j k}(t, \cdot) \frac{\partial^{2}}{\partial x_{j} \partial x_{k}}+b_{j}(t, \cdot) \frac{\partial}{\partial x_{j}} .
$$

Here the coefficients $a_{j k}(t, x)$ are allow to be discontinuous at infinitely many parallel hyper-planes. For more details, see assumptions in Section 3. In fact, there are papers which investigate the well-posedness of martingale problems (or wellposedness of diffusion processes) when the diffusion coefficients $a_{j k}(x)$ are discontinuous in $x$. Some of them can be [2], [3], and [7]. However, they dealt with coefficients $a_{j k}(x)$ which are independent of $t$. We here consider time-dependent coefficients which are discontinuous at hyper-planes $\left\{\left(t, \gamma_{j}, x^{\prime}\right): t \in \mathbb{R}, x^{\prime} \in \mathbb{R}^{d-1}\right\}$, where $\left\{\gamma_{j}\right\}$ is a subset in $\mathbb{R}$ with no limit points. As is shown in [15], the wellposedness of the martingale problem for $L_{t}$ follows from the unique solvability of the corresponding parabolic equation (if the equation is uniquely solvable). Hence our result on parabolic equations in Section 2 plays a major role in the discussion of the martingale problem. For complete details about martingale problems, see the monograph [15].

This paper consists of two sections excluding this introduction. We discuss parabolic equations and martingale problems in Section 2 and 3 respectively.

A few words about notation. We denote by $(t, x)$ a point in $\mathbb{R}^{d+1}$. That is, $(t, x)=\left(t, x_{1}, x^{\prime}\right) \in \mathbb{R} \times \mathbb{R}^{d}=\mathbb{R}^{d+1}$, where $t \in \mathbb{R}, x_{1} \in \mathbb{R}, x^{\prime} \in \mathbb{R}^{d-1}$, and $x \in \mathbb{R}^{d}$. We write $\mathbb{R}_{+}^{d}$ and $\mathbb{R}_{-}^{d}$ for half-spaces

$$
\left\{\left(x_{1}, x^{\prime}\right): x_{1}>0, x^{\prime} \in \mathbb{R}^{d-1}\right\} \quad \text { and } \quad\left\{\left(x_{1}, x^{\prime}\right): x_{1}<0, x^{\prime} \in \mathbb{R}^{d-1}\right\}
$$

respectively. Throughout the paper we set $L_{p}=L_{p}\left(\mathbb{R}^{d+1}\right)$. 
ACKNOWLEDGEMEnTs. The author would like to thank Nicolai V. Krylov for his guidance and support throughout the preparation of this paper.

\section{Second order parabolic equations with discontinuous coefficients}

We consider parabolic equations of the form

$$
\left\{\begin{array}{l}
u_{t}(t, x)=L u(t, x)+f(t, x) \quad \text { in } \quad(0, T) \times \mathbb{R}^{d} \\
u(0, x)=0
\end{array}\right.
$$

where

$$
L u(t, x)=a_{j k}(t, x) u_{x_{j} x_{k}}(t, x)+b_{j}(t, x) u_{x_{j}}(t, x)+c(t, x) u(t, x) .
$$

We prove the existence and uniqueness of solutions to the parabolic equations as above in the Sobolev space $W_{p}^{1,2}\left((0, T) \times \mathbb{R}^{d}\right), 1<p<\infty$. The definition of $W_{p}^{1,2}\left(\left(T_{1}, T_{2}\right) \times \Omega\right)$ can be found in [8] or [9], where $-\infty \leq T_{1}<T_{2} \leq \infty$ and $\Omega$ is an open set in $\mathbb{R}^{d}$.

Let us first state the assumptions on coefficients $a_{j k}(t, x), b_{j}(t, x)$, and $c(t, x)$. Note that in the below the coefficients $a_{j k}(t, x)$ are not uniformly continuous in $x \in \mathbb{R}^{d}$. The function $\omega$ below is an increasing function defined on $[0, \infty)$ such that $\omega(\varepsilon) \rightarrow 0$ as $\varepsilon \searrow 0$.

Assumption 2.1. The coefficients $a_{j k}, j, k=1, \cdots, d$, are defined by

$$
a_{j k}(t, x)=\left\{\begin{array}{llc}
a_{j k}^{+}(t, x) & \text { if } & x_{1}>0 \\
a_{j k}^{-}(t, x) & \text { if } & x_{1}<0
\end{array},\right.
$$

where $a_{j k}^{ \pm}$satisfy the following.

1. $a_{j k}^{+}(t, x)$ are defined on $\overline{\mathbb{R} \times \mathbb{R}_{+}^{d}}$ and $a_{j k}^{-}(t, x)$ are defined on $\overline{\mathbb{R} \times \mathbb{R}_{-}^{d}}$.

2. $a_{j k}^{ \pm}(t, x)=a_{k j}^{ \pm}(t, x)$, and there exists a constant $\kappa \in(0,1)$ such that, for any $t \in \mathbb{R}, x \in \mathbb{R}^{d}$, and $\vartheta \in \mathbb{R}^{d}$,

$$
\kappa|\vartheta|^{2} \leq \sum_{j, k=1}^{d} a_{j k}^{ \pm}(t, x) \vartheta_{j} \vartheta_{k} \leq \kappa^{-1}|\vartheta|^{2} .
$$

3. For each $t \in \mathbb{R}$,

$$
\begin{aligned}
& \left|a_{j k}^{+}(t, x)-a_{j k}^{+}(t, y)\right| \leq \omega(|x-y|) \quad \text { for } \quad x, y \in \overline{\mathbb{R}_{+}^{d}}, \\
& \left|a_{j k}^{-}(t, x)-a_{j k}^{-}(t, y)\right| \leq \omega(|x-y|) \quad \text { for } \quad x, y \in \overline{\mathbb{R}_{-}^{d}} .
\end{aligned}
$$


4. For $t, s \in \mathbb{R}$ and $x^{\prime}, y^{\prime} \in \mathbb{R}^{d-1}$,

$$
\begin{aligned}
& \left|a_{j k}^{+}\left(t, 0, x^{\prime}\right)-a_{j k}^{+}\left(s, 0, y^{\prime}\right)\right| \leq \omega\left(|t-s|+\left|x^{\prime}-y^{\prime}\right|\right), \\
& \left|a_{j k}^{-}\left(t, 0, x^{\prime}\right)-a_{j k}^{-}\left(s, 0, y^{\prime}\right)\right| \leq \omega\left(|t-s|+\left|x^{\prime}-y^{\prime}\right|\right) .
\end{aligned}
$$

Assumption 2.2. The coefficients $b_{j}(t, x)$ and $c(t, x)$ are measurable functions defined on $\mathbb{R} \times \mathbb{R}^{d}$ satisfying

$$
\left|b_{j}(t, x)\right|+|c(t, x)| \leq K
$$

for some positive constant $K$.

The first case we consider is

$$
u_{t}(t, x)=L_{0} u(t, x)-\lambda u(t, x)+f(t, x) \text { in } \quad \mathbb{R} \times \mathbb{R}^{d}
$$

with

$$
L_{0}=\left\{\begin{array}{lll}
L_{0}^{+}=a_{j k}^{+} D_{j k} & \text { in } & \mathbb{R} \times \mathbb{R}_{+}^{d} \\
L_{0}^{-}=a_{j k}^{-} D_{j k} & \text { in } & \mathbb{R} \times \mathbb{R}_{-}^{d}
\end{array},\right.
$$

where $a_{j k}^{+}$and $a_{j k}^{-}$are constant. Recall that $L_{p}=L_{p}\left(\mathbb{R}^{d+1}\right)$.

Theorem 2.3. For any $\lambda \geq 0$ and $u \in C_{0}^{\infty}\left(\mathbb{R}^{d+1}\right)$,

$$
\lambda\|u\|_{L_{p}}+\left\|u_{t}\right\|_{L_{p}}+\left\|u_{x x}\right\|_{L_{p}} \leq N\left\|L_{0} u-\lambda u-u_{t}\right\|_{L_{p}},
$$

where $N$ depends only on $d, p$, and $\kappa$.

To prove this theorem we need the following function spaces and trace theorem which can be found in $[8,17]$. For a non-negative real number $l$, let

$$
W_{p}^{l / 2, l}\left(\mathbb{R} \times \mathbb{R}^{d-1}\right)=L_{p}\left(\mathbb{R}, W_{p}^{l}\left(\mathbb{R}^{d-1}\right)\right) \cap W_{p}^{l / 2}\left(\mathbb{R}, L_{p}\left(\mathbb{R}^{d-1}\right)\right),
$$

where $W_{p}^{s}\left(\mathbb{R}^{d-1}\right), s=0,1,2, \cdots$, are Sobolev spaces and $W_{p}^{s}\left(\mathbb{R}^{d-1}\right), s \neq$ integer, $s>0$, are Slobodeckij spaces.

Theorem 2.4. Set $l=2-1 / p$. There exists the trace operator $T$ from

$$
W_{p}^{1,2}\left(\mathbb{R} \times \mathbb{R}_{+}^{d}\right) \quad \text { onto } \quad W_{p}^{l / 2, l}\left(\mathbb{R} \times \mathbb{R}^{d-1}\right) \times W_{p}^{(l-1) / 2, l-1}\left(\mathbb{R} \times \mathbb{R}^{d-1}\right)
$$

such that

$$
T u=\left(u\left(t, 0, x^{\prime}\right), u_{x_{1}}\left(t, 0, x^{\prime}\right)\right)
$$

and

$$
\|u(\cdot, 0, \cdot)\|_{W_{p}^{l / 2, l}(\Gamma)}+\left\|u_{x_{1}}(\cdot, 0, \cdot)\right\|_{W_{p}^{(l-1) / 2, l-1}(\Gamma)} \leq N\|u\|_{W_{p}^{1,2}\left(\mathbb{R} \times \mathbb{R}_{+}^{d}\right)}
$$

for $u \in W_{p}^{1,2}\left(\mathbb{R} \times \mathbb{R}_{+}^{d}\right)$, where $\Gamma=\mathbb{R} \times \mathbb{R}^{d-1}$ and $N$ depends only on $d$ and $p$. Moreover, $T$ has a bounded linear right inverse (extension operator). 
We also need the following notations, similar to those in [10, 12]. Set

$$
\begin{gathered}
\alpha^{ \pm}\left(\xi^{\prime}\right)=\mathrm{i} \sum_{j=2}^{d} a_{1 j}^{ \pm} \xi_{j}, \quad \xi^{\prime}=\left(\xi_{2}, \cdots, \xi_{d}\right) \in \mathbb{R}^{d-1}, \quad \mathrm{i}=\sqrt{-1} \\
\beta^{ \pm}\left(\xi^{\prime}\right)=\sum_{j, k=2}^{d} a_{j k}^{ \pm} \xi_{j} \xi_{k}+1,
\end{gathered}
$$

and

$$
\begin{gathered}
H^{ \pm}\left(\xi^{\prime}\right)=\left(\alpha^{ \pm}\left(\xi^{\prime}\right)\right)^{2}+a_{11}^{ \pm} \beta^{ \pm}\left(\xi^{\prime}\right) \\
=-\left(\sum_{j=2}^{d} a_{1 j}^{ \pm} \xi_{j}\right)^{2}+a_{11}^{ \pm}\left(\sum_{j, k=2}^{d} a_{j k}^{ \pm} \xi_{j} \xi_{k}+1\right) .
\end{gathered}
$$

Let $s \in \mathbb{R}$. We denote

$$
\begin{aligned}
z^{+}\left(s, \xi^{\prime}\right) & =\frac{-\alpha^{+}\left(\xi^{\prime}\right)-\sqrt{H^{+}\left(\xi^{\prime}\right)+\mathrm{i} a_{11}^{+} s}}{a_{11}^{+}}, \\
z^{-}\left(s, \xi^{\prime}\right) & =\frac{-\alpha^{-}\left(\xi^{\prime}\right)+\sqrt{H^{-}\left(\xi^{\prime}\right)+\mathrm{i} a_{11}^{-} s}}{a_{11}^{-}}, \\
z\left(s, \xi^{\prime}\right) & =z^{+}\left(s, \xi^{\prime}\right)-z^{-}\left(s, \xi^{\prime}\right),
\end{aligned}
$$

where $\sqrt{w}$ means, from now on, that branch of the analytic function $w^{1 / 2}$ that has non-negative real part. Note that

$$
\mathfrak{R}\left[z^{+}\left(s, \xi^{\prime}\right)\right]=-\frac{1}{a_{11}^{+}} \mathfrak{l}\left[\sqrt{H^{+}\left(\xi^{\prime}\right)+\mathrm{i} a_{11}^{+} s}\right]
$$

and

$$
\mathfrak{R}\left[z^{-}\left(s, \xi^{\prime}\right)\right]=\frac{1}{a_{11}^{-}} \mathfrak{R}\left[\sqrt{H^{-}\left(\xi^{\prime}\right)+\mathrm{i} a_{11}^{-} s}\right] .
$$

The following estimates hold true for $\Re\left[z^{ \pm}\left(s, \xi^{\prime}\right)\right]$. One can find the same estimates in [12].

\section{Lemma 2.5.}

$$
-N_{2} \sqrt{1+\left|\xi^{\prime}\right|^{2}+|s|} \leq \Re\left[z^{+}\left(s, \xi^{\prime}\right)\right] \leq-N_{1} \sqrt{1+\left|\xi^{\prime}\right|^{2}+|s|}
$$

and

$$
N_{1} \sqrt{1+\left|\xi^{\prime}\right|^{2}+|s|} \leq \Re\left[z^{-}\left(s, \xi^{\prime}\right)\right] \leq N_{2} \sqrt{1+\left|\xi^{\prime}\right|^{2}+|s|},
$$

where $N_{1}$ and $N_{2}$ depend only on $\kappa$. 
Proof. The lemma follows from the fact that $\kappa \leq a_{11}^{ \pm} \leq \kappa^{-1}$ and

$$
N_{1}\left|\xi^{\prime}\right|^{2} \leq H^{ \pm}\left(\xi^{\prime}\right)-a_{11}^{ \pm} \leq N_{2}\left|\xi^{\prime}\right|^{2}
$$

which is proved in Lemma 3 in [10]. Here the constants $N_{1}$ and $N_{2}$ depend only on $\kappa$.

The main step in the proof of Theorem 2.3 is to prove that an operator $\mathcal{T}$ defined by

$$
\widetilde{\mathcal{T} h}=\widetilde{h} / z\left(s, \xi^{\prime}\right), \quad h \in C_{0}^{\infty}\left(\mathbb{R} \times \mathbb{R}^{d-1}\right),
$$

can be extended to a bounded operator from

$$
W_{p}^{(l-1) / 2, l-1}\left(\mathbb{R} \times \mathbb{R}^{d-1}\right) \quad \text { into } \quad W_{p}^{l / 2, l}\left(\mathbb{R} \times \mathbb{R}^{d-1}\right),
$$

where $l=2-1 / p$ and $\mathbb{R} \times \mathbb{R}^{d-1}=\left\{\left(t, 0, x^{\prime}\right): t \in \mathbb{R}, x^{\prime} \in \mathbb{R}^{d-1}\right\}$. Here $\tilde{f}$ is the Fourier transform of $f$ in $\mathbb{R} \times \mathbb{R}^{d-1}$. To prove this, we start with a lemma showing that $\left(1+s^{2}\right)^{1 / 4} / z\left(s, \xi^{\prime}\right)$ and $\mathrm{i} \xi_{j} / z\left(s, \xi^{\prime}\right)$ are multipliers. Note that we sometimes mean by $\mathbb{R}^{d}$, especially in Lemma 2.6 and 2.7 , the space $\left\{\left(t, 0, x^{\prime}\right): t \in \mathbb{R}, x^{\prime} \in\right.$ $\left.\mathbb{R}^{d-1}\right\}$.

\section{Lemma 2.6.}

$$
\frac{\left(1+s^{2}\right)^{1 / 4}}{z\left(s, \xi^{\prime}\right)} \quad \text { and } \quad \frac{\mathrm{i} \xi_{j}}{z\left(s, \xi^{\prime}\right)}, \quad j=2, \cdots, d \text {, }
$$

are multipliers for $L_{p}\left(\mathbb{R}^{d}\right)$. That is, if we set $\widetilde{\mathcal{T} f}\left(s, \xi^{\prime}\right)=m\left(s, \xi^{\prime}\right) \widetilde{f}$, where $f \in$ $L_{2}\left(\mathbb{R}^{d}\right) \cap L_{p}\left(\mathbb{R}^{d}\right)$ and $m\left(s, \xi^{\prime}\right)$ is either of the above multipliers, then we have

$$
\|\mathcal{T} f\|_{L_{p}\left(\mathbb{R}^{d}\right)} \leq N\|f\|_{L_{p}\left(\mathbb{R}^{d}\right)},
$$

where $N$ depends only on $d, p$, and $\kappa$.

Proof. Set $s=\xi_{1}$ (recall that $\left.\xi^{\prime}=\left(\xi_{2}, \cdots, \xi_{d}\right)\right)$. We prove

$$
\left|\frac{\partial^{m}}{\partial \xi_{j_{1}} \cdots \partial \xi_{j_{m}}}\left(\frac{\left(1+\xi_{1}^{2}\right)^{1 / 4}}{z\left(\xi_{1}, \xi^{\prime}\right)}\right)\right| \leq \frac{N}{\left|\xi_{j_{1}} \cdots \xi_{j_{m}}\right|}
$$

and

$$
\left|\frac{\partial^{m}}{\partial \xi_{j_{1}} \cdots \partial \xi_{j_{m}}}\left(\frac{\mathrm{i} \xi_{j}}{z\left(\xi_{1}, \xi^{\prime}\right)}\right)\right| \leq \frac{N}{\left|\xi_{j_{1}} \cdots \xi_{j_{m}}\right|}, \quad j=2, \cdots, d,
$$

where $m=1, \cdots, d, j_{k} \in\{1, \cdots, d\}$, and $j_{k} \neq j_{l}$ if $k \neq l$. Especially, the constant $N$ depends only on $d$ and $\kappa$. Once these inequalities are proved, the lemma follows from Mikhlin's theorem (Theorem 6' on page 109 [14] or see page 289 [8]). 
First, we prove that, for a multi-index $\alpha, \alpha=\left(0, \alpha_{2}, \cdots, \alpha_{d}\right)$,

$$
\left|D^{\alpha} \sqrt{H^{ \pm}\left(\xi^{\prime}\right)+\mathrm{i} a_{11}^{ \pm} \xi_{1}}\right| \leq N\left(1+\left|\xi^{\prime}\right|^{2}+\left|\xi_{1}\right|\right)^{-\frac{|\alpha|-1}{2}},
$$

where $N$ depends only on $d, \alpha$, and $\kappa$. Using the matrix $\sqrt{\left[a_{j k}^{+}\right]}$and an appropriate orthogonal change of variables, we can find a matrix $A=\left[\sigma_{j k}\right]$ satisfying the following:

(i) The mapping $x \rightarrow A x$ is a diffeomorphism from $\mathbb{R}_{+}^{d}$ onto itself.

(ii) For a twice differentiable function $v(x)$ defined on $\mathbb{R}_{+}^{d}$, set $w(x)=v(A x)$. Then $L_{0}^{+} v(A x)=\Delta w(x)$ in $\mathbb{R}_{+}^{d}$, where, as we recall, $L_{0}^{+} v=a_{j k}^{+} v_{x_{j} x_{k}}$.

Notice that from the above two properties of $A=\left[\sigma_{j k}\right]$ we have $a_{j k}^{+}=\sum_{l=1}^{d} \sigma_{j l} \sigma_{k l}$, $k=1, \cdots, d$, and $\sigma_{1 k}=0, k=2, \cdots, d$. Denote by $B$ the sub-matrix formed by removing the first row and column of $A$. Then $B$ is a non-singular matrix. Observe that

$$
\begin{aligned}
\left|B^{*} \xi^{\prime}\right|^{2} & =\sum_{j, k=2}^{d}\left(a_{j k}^{+}-\sigma_{j 1} \sigma_{k 1}\right) \xi_{j} \xi_{k}=-\sum_{j, k=2}^{d} \sigma_{j 1} \sigma_{k 1} \xi_{j} \xi_{k}+\sum_{j, k=2}^{d} a_{j k}^{+} \xi_{j} \xi_{k} \\
& =-\left(\sum_{j=2}^{d} \sigma_{j 1} \xi_{j}\right)^{2}+\sum_{j, k=2}^{d} a_{j k}^{+} \xi_{j} \xi_{k}=-\frac{1}{\sigma_{11}^{2}}\left(\sum_{j=2}^{d} \sigma_{11} \sigma_{j 1} \xi_{j}\right)^{2}+\sum_{j, k=2}^{d} a_{j k}^{+} \xi_{j} \xi_{k} \\
& =-\frac{1}{a_{11}^{+}}\left(\sum_{j=2}^{d} a_{1 j}^{+} \xi_{j}\right)^{2}+\sum_{j, k=2}^{d} a_{j k}^{+} \xi_{j} \xi_{k}=\frac{1}{a_{11}^{+}} H^{+}\left(\xi^{\prime}\right)-1 .
\end{aligned}
$$

Thus

$$
\sqrt{H^{+}\left(\xi^{\prime}\right)+\mathrm{i} a_{11}^{+} \xi_{1}}=\sqrt{a_{11}^{+}\left(\left|B^{*} \xi^{\prime}\right|^{2}+1+\mathrm{i} \xi_{1}\right)} .
$$

From this and (2.3) we see that

$$
\left|D^{\alpha} \sqrt{H^{+}\left(\xi^{\prime}\right)+\mathrm{i} a_{11}^{+} \xi_{1}}\right| \leq N\left(1+\left|\xi^{\prime}\right|^{2}+\left|\xi_{1}\right|\right)^{-\frac{|\alpha|-1}{2}},
$$

where $N$ depends only on $d, \alpha$, and $\kappa$. By the same calculation, we also have the above inequality with $H^{-}\left(\xi^{\prime}\right)$ and $a_{11}^{-}$in place of $H^{+}\left(\xi^{\prime}\right)$ and $a_{11}^{+}$. Therefore, (2.6) is proved. Moreover, it follows easily that

$$
\left|D^{\alpha}\left[\left(H^{ \pm}\left(\xi^{\prime}\right)+\mathrm{i} a_{11}^{ \pm} \xi_{1}\right)^{-1 / 2}\right]\right| \leq N\left(1+\left|\xi^{\prime}\right|^{2}+\left|\xi_{1}\right|\right)^{-\frac{|\alpha|+1}{2}} .
$$


Next, we prove that, for $\alpha=\left(0, \alpha_{2}, \cdots, \alpha_{d}\right)$,

$$
\left|D^{\alpha}\left(\frac{1}{z\left(\xi_{1}, \xi^{\prime}\right)}\right)\right| \leq N\left(1+\left|\xi^{\prime}\right|^{2}+\left|\xi_{1}\right|\right)^{-\frac{|\alpha|+1}{2}},
$$

where $N$ depends only on $d, \alpha$, and $\kappa$. If $\alpha=0$, then by Lemma 2.5

$$
\left|\frac{1}{z\left(\xi_{1}, \xi^{\prime}\right)}\right| \leq \frac{1}{-\Re\left[z^{+}\left(\xi_{1}, \xi^{\prime}\right)\right]+\Re\left[z^{-}\left(\xi_{1}, \xi^{\prime}\right)\right]} \leq N\left(1+\left|\xi^{\prime}\right|^{2}+\left|\xi_{1}\right|\right)^{-\frac{1}{2}}
$$

where $N$ depends only on $\kappa$. For a multi-index $|\alpha| \geq 1$, the inequality (2.8) is justified using (2.6) and induction.

Finally, we prove that, for $\alpha=\left(0, \alpha_{2}, \cdots, \alpha_{d}\right)$,

$$
\left|D^{\alpha}\left[\frac{\partial}{\partial \xi_{1}}\left(\frac{1}{z\left(\xi_{1}, \xi^{\prime}\right)}\right)\right]\right| \leq N\left(1+\left|\xi^{\prime}\right|^{2}+\left|\xi_{1}\right|\right)^{-\frac{|\alpha|+3}{2}},
$$

where $N$ depends only on $d, \alpha$, and $\kappa$. This follows from (2.7), (2.8), and

$$
\frac{\partial}{\partial \xi_{1}}\left(\frac{1}{z\left(\xi_{1}, \xi^{\prime}\right)}\right)=\frac{\mathrm{i}}{2 z\left(\xi_{1}, \xi^{\prime}\right)^{2}}\left(\frac{a_{11}^{+}}{\sqrt{H^{+}\left(\xi^{\prime}\right)+\mathrm{i} a_{11}^{+} \xi_{1}}}+\frac{a_{11}^{-}}{\sqrt{H^{-}\left(\xi^{\prime}\right)+\mathrm{i} a_{11}^{-} \xi_{1}}}\right) .
$$

Now we use (2.8) and (2.9) to prove (2.4) and (2.5). For example, if $\xi_{j_{1}}=\xi_{1}$ and $D^{\alpha}=\frac{\partial^{m-1}}{\partial \xi_{j_{2}} \cdots \partial \xi_{j m}}$, then

$$
\begin{aligned}
& \left|\frac{\partial^{m}}{\partial \xi_{j_{1}} \cdots \partial \xi_{j_{m}}}\left(\frac{\left(1+\xi_{1}^{2}\right)^{1 / 4}}{z\left(\xi_{1}, \xi^{\prime}\right)}\right)\right| \\
& =\left|\frac{\xi_{1}}{2\left(1+\xi_{1}^{2}\right)^{3 / 4}} D^{\alpha}\left(\frac{1}{z\left(\xi_{1}, \xi^{\prime}\right)}\right)+\left(1+\xi_{1}^{2}\right)^{1 / 4} D^{\alpha}\left[\frac{\partial}{\partial \xi_{1}}\left(\frac{1}{z\left(\xi_{1}, \xi^{\prime}\right)}\right)\right]\right| \\
& \leq N\left(\frac{\left|\xi_{1}\right|}{\left(1+\xi_{1}^{2}\right)^{3 / 4}\left(1+\left|\xi^{\prime}\right|^{2}+\left|\xi_{1}\right|\right)^{(|\alpha|+1) / 2}}+\frac{\left(1+\xi_{1}^{2}\right)^{1 / 4}}{\left(1+\left|\xi^{\prime}\right|^{2}+\left|\xi_{1}\right|\right)^{(|\alpha|+3) / 2}}\right) \\
& \leq \frac{N}{\left|\xi_{1}\right|\left(1+\left|\xi^{\prime}\right|^{2}+\left|\xi_{1}\right|\right)^{\frac{|\alpha|}{2}}} \leq \frac{N}{\left|\xi_{j_{1}} \cdots \xi_{j_{m}}\right|} .
\end{aligned}
$$

The other cases are proved in a similar way. The lemma is proved.

Using the above result and interpolation, we prove the following lemma. 
Lemma 2.7. Let $\mathcal{T}$ be an operator such that

$$
\widetilde{\mathcal{T} h}=\frac{1}{z\left(s, \xi^{\prime}\right)} \tilde{h}, \quad h \in C_{0}^{\infty}\left(\mathbb{R}^{d}\right),
$$

where $\widetilde{\mathcal{T} h}$ and $\tilde{h}$ are Fourier transforms of $\mathcal{T} h$ and $h$ in $\mathbb{R}^{d}$ respectively. Set $l=$ $2-1 / p$, then

$$
\|\mathcal{T} h\|_{W_{p}^{l / 2, l}\left(\mathbb{R} \times \mathbb{R}^{d-1}\right)} \leq N\|h\|_{W_{p}^{(l-1) / 2, l-1}\left(\mathbb{R} \times \mathbb{R}^{d-1}\right)},
$$

where $N$ depends only on $d, p$, and $\kappa$.

Proof. Define an operator $\mathcal{S}$ by

$$
\widetilde{\mathcal{S} h}=\frac{\left(1+s^{2}\right)^{1 / 4}}{z\left(s, \xi^{\prime}\right)} \widetilde{h}
$$

where $h \in C_{0}^{\infty}\left(\mathbb{R}^{d}\right)$. By Lemma 2.6 the operator $\mathcal{S}$ can be extended to a bounded operator from $L_{p}\left(\mathbb{R}, L_{p}\left(\mathbb{R}^{d-1}\right)\right)$ to $L_{p}\left(\mathbb{R}, L_{p}\left(\mathbb{R}^{d-1}\right)\right)$ and from $W_{p}^{1}\left(\mathbb{R}, L_{p}\left(\mathbb{R}^{d-1}\right)\right)$ to $W_{p}^{1}\left(\mathbb{R}, L_{p}\left(\mathbb{R}^{d-1}\right)\right)$. Here we used the fact that $\mathcal{S}\left(D^{\alpha} h\right)=D^{\alpha} \mathcal{S}(h)$. By real interpolation (see [1]) we have

$$
\left(L_{p}\left(\mathbb{R}, L_{p}\left(\mathbb{R}^{d-1}\right)\right), W_{p}^{1}\left(\mathbb{R}, L_{p}\left(\mathbb{R}^{d-1}\right)\right)_{(l-1) / 2, p}=W_{p}^{(l-1) / 2}\left(\mathbb{R}, L_{p}\left(\mathbb{R}^{d-1}\right)\right) .\right.
$$

Thus

$$
\|\mathcal{S} h\|_{W_{p}^{(l-1) / 2}\left(\mathbb{R}, L_{p}\left(\mathbb{R}^{d-1}\right)\right)} \leq N\|h\|_{W_{p}^{(l-1) / 2}\left(\mathbb{R}, L_{p}\left(\mathbb{R}^{d-1}\right)\right)},
$$

where $N$ depends only on $d, p$, and $\kappa$. Since $\left(1+s^{2}\right)^{1 / 4}$ is an isometric multiplier from $W_{p}^{l / 2}(\mathbb{R})\left(=B_{p, p}^{l / 2}(\mathbb{R})\right)$ to $W_{p}^{(l-1) / 2}(\mathbb{R})\left(=B_{p, p}^{(l-1) / 2}(\mathbb{R})\right)$,

$$
\begin{gathered}
\|\mathcal{T} h\|_{W_{p}^{l / 2}\left(\mathbb{R}, L_{p}\left(\mathbb{R}^{d-1}\right)\right)}^{p}=\int_{\mathbb{R}^{d-1}}\left\|\mathcal{T} h\left(\cdot, x^{\prime}\right)\right\|_{W_{p}^{l / 2}(\mathbb{R})}^{p} d x^{\prime} \\
=\int_{\mathbb{R}^{d-1}}\left\|\mathcal{F}_{1}^{-1}\left(1+s^{2}\right)^{1 / 4} \mathcal{F}_{1}(\mathcal{T} h)\left(\cdot, x^{\prime}\right)\right\|_{W_{p}^{(l-1) / 2}(\mathbb{R})}^{p} d x^{\prime} \\
=\|\mathcal{S} h\|_{W_{p}^{(l-1) / 2}\left(\mathbb{R}, L_{p}\left(\mathbb{R}^{d-1}\right)\right)}^{p}
\end{gathered}
$$

where $\mathcal{F}_{1}$ and $\mathcal{F}_{1}^{-1}$ are the Fourier transform and its inverse on $\mathbb{R}$. This and the inequality (2.11) prove

$$
\|\mathcal{T} h\|_{W_{p}^{l / 2}\left(\mathbb{R}, L_{p}\left(\mathbb{R}^{d-1}\right)\right)} \leq N\|h\|_{W_{p}^{(l-1) / 2}\left(\mathbb{R}, L_{p}\left(\mathbb{R}^{d-1}\right)\right)},
$$

where $N$ depends only on $d, p$, and $\kappa$. 
On the other hand, from Lemma 2.6, we know that $\mathrm{i} \xi_{j} / z\left(s, \xi^{\prime}\right), j=2, \cdots, d$, are multipliers for $L_{p}\left(\mathbb{R}^{d}\right)$. This implies that

$$
\int_{-\infty}^{\infty}\|\mathcal{T} h(t, \cdot)\|_{W_{p}^{k+1}\left(\mathbb{R}^{d-1}\right)}^{p} d t \leq N \int_{-\infty}^{\infty}\|h(t, \cdot)\|_{W_{p}^{k}\left(\mathbb{R}^{d-1}\right)}^{p} d t,
$$

where $k=0,1$ and $N$ depends only on $d, p$, and $\kappa$. Hence $\mathcal{T}$ can be extended to a bounded operator from

$$
L_{p}\left(\mathbb{R}, W_{p}^{k}\left(\mathbb{R}^{d-1}\right)\right) \quad \text { to } \quad L_{p}\left(\mathbb{R}, W_{p}^{k+1}\left(\mathbb{R}^{d-1}\right)\right),
$$

where $k=0,1$. Note that by real interpolation (see [1] or Theorem 1.18.4 in [16]), we have

$$
\left(L_{p}\left(\mathbb{R}, L_{p}\left(\mathbb{R}^{d-1}\right)\right), L_{p}\left(\mathbb{R}, W_{p}^{1}\left(\mathbb{R}^{d-1}\right)\right)\right)_{l-1, p}=L_{p}\left(\mathbb{R}, W_{p}^{l-1}\left(\mathbb{R}^{d-1}\right)\right)
$$

and

$$
\left(L_{p}\left(\mathbb{R}, W_{p}^{1}\left(\mathbb{R}^{d-1}\right)\right), L_{p}\left(\mathbb{R}, W_{p}^{2}\left(\mathbb{R}^{d-1}\right)\right)\right)_{l-1, p}=L_{p}\left(\mathbb{R}, W_{p}^{l}\left(\mathbb{R}^{d-1}\right)\right) .
$$

Therefore,

$$
\|\mathcal{T} h\|_{L_{p}\left(\mathbb{R}, W_{p}^{l}\left(\mathbb{R}^{d-1}\right)\right)} \leq N\|h\|_{L_{p}\left(\mathbb{R}, W_{p}^{l-1}\left(\mathbb{R}^{d-1}\right)\right)},
$$

where $N$ depends only on $d, p$, and $\kappa$.

Finally, recall that

$$
W_{p}^{s / 2, s}\left(\mathbb{R} \times \mathbb{R}^{d-1}\right)=L_{p}\left(\mathbb{R}, W_{p}^{s}\left(\mathbb{R}^{d-1}\right)\right) \cap W_{p}^{s / 2}\left(\mathbb{R}, L_{p}\left(\mathbb{R}^{d-1}\right)\right),
$$

where $s$ is either $l$ or $l-1$. From this, (2.12), and (2.13), the inequality (2.10) follows.

We need the Fourier transform of a solution to a parabolic equation defined on a parabolic half-space (e.g. $\mathbb{R} \times \mathbb{R}_{+}^{d}$ ).

Lemma 2.8. For $g\left(t, x^{\prime}\right) \in C_{0}^{\infty}\left(\mathbb{R} \times \mathbb{R}^{d-1}\right)\left(=C_{0}^{\infty}\left(\mathbb{R}^{d}\right)\right)$, let $v^{+} \in W_{p}^{1,2}\left(\mathbb{R} \times \mathbb{R}_{+}^{d}\right)$ and $v^{-} \in W_{p}^{1,2}\left(\mathbb{R} \times \mathbb{R}_{-}^{d}\right)$ be the solutions of the equations

$$
\left\{\begin{array}{l}
v_{t}^{+}=L_{0}^{+} v^{+}-v^{+} \text {in } \mathbb{R} \times \mathbb{R}_{+}^{d} \\
v^{+}\left(t, 0, x^{\prime}\right)=g\left(t, x^{\prime}\right)
\end{array}, \quad\left\{\begin{array}{l}
v_{t}^{-}=L_{0}^{-} v^{-}-v^{-} \text {in } \mathbb{R} \times \mathbb{R}_{-}^{d} \\
v^{-}\left(t, 0, x^{\prime}\right)=g\left(t, x^{\prime}\right) .
\end{array}\right.\right.
$$

Then

$$
\widetilde{v_{x_{1}}^{+}}\left(s, 0, \xi^{\prime}\right)=z^{+}\left(s, \xi^{\prime}\right) \widetilde{g}\left(s, \xi^{\prime}\right) \text { and } \widetilde{v_{x_{1}}^{-}}\left(s, 0, \xi^{\prime}\right)=z^{-}\left(s, \xi^{\prime}\right) \widetilde{g}\left(s, \xi^{\prime}\right),
$$

where Fourier transforms are taken with respect to $\left(t, x^{\prime}\right) \in \mathbb{R} \times \mathbb{R}^{d-1}$. 
Proof. First consider

$$
\left\{\begin{array}{l}
v_{t}=\Delta v-v \text { in } \mathbb{R} \times \mathbb{R}_{+}^{d} \\
v\left(t, 0, x^{\prime}\right)=g\left(t, x^{\prime}\right)
\end{array}\right.
$$

where $g \in C_{0}^{\infty}\left(\mathbb{R} \times \mathbb{R}^{d-1}\right)$. Let

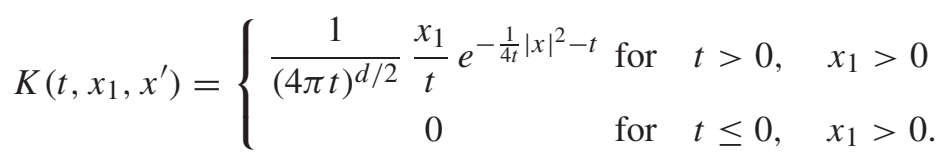

Then the solution to (2.14) is

$$
v\left(t, x_{1}, x^{\prime}\right)=\left[K\left(\cdot, x_{1}, \cdot\right) * g(\cdot, \cdot)\right]\left(t, x^{\prime}\right) .
$$

We see that

$$
\int_{\mathbb{R}^{d}} e^{-\mathrm{i}\left(s t+\xi^{\prime} \cdot x^{\prime}\right)} K\left(t, x_{1}, x^{\prime}\right) d t d x^{\prime}=e^{-x_{1} \sqrt{1+\left|\xi^{\prime}\right|^{2}+\mathrm{i} s}} .
$$

Thus we have

$$
\widetilde{v}\left(s, x_{1}, \xi^{\prime}\right)=\widetilde{g}\left(s, \xi^{\prime}\right) e^{-x_{1} \sqrt{1+\left|\xi^{\prime}\right|^{2}+\mathrm{i} s}}
$$

and

$$
\widetilde{v_{x_{1}}}\left(s, 0, \xi^{\prime}\right)=-\widetilde{g}\left(s, \xi^{\prime}\right) \sqrt{1+\left|\xi^{\prime}\right|^{2}+\mathrm{i} s},
$$

where the second equality can be justified as follows. Note that

$$
v\left(t, x_{1}, x^{\prime}\right)=c_{d} \int_{\mathbb{R}^{d}} e^{\mathrm{i}\left(t s+x^{\prime} \cdot \xi^{\prime}\right)} \widetilde{g}\left(s, \xi^{\prime}\right) e^{-x_{1} \sqrt{1+\left|\xi^{\prime}\right|^{2}+\mathrm{i} s}} d s d \xi^{\prime},
$$

where $c_{d}=(2 \pi)^{-d / 2}$. Thus

$$
\begin{aligned}
v_{x_{1}}\left(t, 0, x^{\prime}\right) & =\lim _{h \rightarrow 0} c_{d} \int_{\mathbb{R}^{d}} e^{\mathrm{i}\left(t s+x^{\prime} \cdot \xi^{\prime}\right)} \widetilde{g}\left(s, \xi^{\prime}\right) \frac{e^{-h \sqrt{1+\left|\xi^{\prime}\right|^{2}+\mathrm{i} s}}-1}{h} d s d \xi^{\prime} \\
& =-c_{d} \int_{\mathbb{R}^{d}} e^{\mathrm{i}\left(t s+x^{\prime} \cdot \xi^{\prime}\right)} \tilde{g}\left(s, \xi^{\prime}\right) \sqrt{1+\left|\xi^{\prime}\right|^{2}+\mathrm{i} s} d s d \xi^{\prime}
\end{aligned}
$$

By taking Fourier transforms, we arrive at (2.15). This, together with the definition of $z^{+}\left(s, \xi^{\prime}\right)$, proves the case $L_{0}^{+}=\Delta$. The case $L_{0}^{-}=\Delta$ is proved similarly. For general $L_{0}^{+}$and $L_{0}^{-}$, we use the change of variables in the proof of Lemma 2.6. 
Proof of Theorem 2.3. We prove the inequality (2.1) only for $\lambda>0$ because the inequality with $\lambda=0$ is obtained by continuity (with respect to $\lambda$ ). In the case $\lambda>$ 0 , since we can use a dilation argument, it is enough to prove (i.e., the inequality (2.1) with $\lambda=1$ )

$$
\|u\|_{W_{p}^{1,2}\left(\mathbb{R} \times \mathbb{R}^{d}\right)} \leq N\left\|L_{0} u-u-u_{t}\right\|_{L_{p}},
$$

where $N$ depends only on $d, p$, and $\kappa$.

Let $f=u_{t}+u-L_{0} u$ and $g\left(t, x^{\prime}\right)=u\left(t, 0, x^{\prime}\right)$. Consider

$$
\left\{\begin{array} { l } 
{ v _ { t } ^ { + } = L _ { 0 } ^ { + } v ^ { + } - v ^ { + } \text { in } \mathbb { R } \times \mathbb { R } _ { + } ^ { d } } \\
{ v ^ { + } ( t , 0 , x ^ { \prime } ) = g ( t , x ^ { \prime } ) , }
\end{array} \left\{\begin{array}{l}
w_{t}^{+}=L_{0}^{+} w^{+}-w^{+}+f \text { in } \mathbb{R} \times \mathbb{R}_{+}^{d} \\
w^{+}\left(t, 0, x^{\prime}\right)=0,
\end{array}\right.\right.
$$

and

$$
\left\{\begin{array} { l } 
{ v _ { t } ^ { - } = L _ { 0 } ^ { - } v ^ { - } - v ^ { - } \text { in } \mathbb { R } \times \mathbb { R } _ { - } ^ { d } } \\
{ v ^ { - } ( t , 0 , x ^ { \prime } ) = g ( t , x ^ { \prime } ) , }
\end{array} \left\{\begin{array}{l}
w_{t}^{-}=L_{0}^{-} w^{-}-w^{-}+f \text { in } \mathbb{R} \times \mathbb{R}_{-}^{d} \\
w^{-}\left(0, t, x^{\prime}\right)=0 .
\end{array}\right.\right.
$$

As is well known, the above equations have unique solutions

$$
v^{+}, w^{+} \in W_{p}^{1,2}\left(\mathbb{R} \times \mathbb{R}_{+}^{d}\right), \quad v^{-}, w^{-} \in W_{p}^{1,2}\left(\mathbb{R} \times \mathbb{R}_{-}^{d}\right)
$$

satisfying (we set $l=2-1 / p$.)

$\left\|v^{+}\right\|_{W_{p}^{1,2}\left(\mathbb{R} \times \mathbb{R}_{+}^{d}\right)} \leq N\|g\|_{W_{p}^{l / 2, l}\left(\mathbb{R} \times \mathbb{R}^{d-1}\right)},\left\|w^{+}\right\|_{W_{p}^{1,2}\left(\mathbb{R} \times \mathbb{R}_{+}^{d}\right)} \leq N\|f\|_{L_{p}\left(\mathbb{R} \times \mathbb{R}_{+}^{d}\right)}$,

and

$\left\|v^{-}\right\|_{W_{p}^{1,2}\left(\mathbb{R} \times \mathbb{R}_{-}^{d}\right)} \leq N\|g\|_{W_{p}^{l / 2, l}\left(\mathbb{R} \times \mathbb{R}^{d-1}\right)},\left\|w^{-}\right\|_{W_{p}^{1,2}\left(\mathbb{R} \times \mathbb{R}_{-}^{d}\right)} \leq N\|f\|_{L_{p}\left(\mathbb{R} \times \mathbb{R}_{-}^{d}\right)}$,

where $N$ depends only on $d, p$, and $\kappa$. We see that

$$
u=\left\{\begin{array}{lll}
v^{+}+w^{+} & \text {in } & \mathbb{R} \times \mathbb{R}_{+}^{d} \\
v^{-}+w^{-} & \text {in } & \mathbb{R} \times \mathbb{R}_{-}^{d}
\end{array} .\right.
$$

It then follows that

$$
\|u\|_{W_{p}^{1,2}\left(\mathbb{R} \times \mathbb{R}^{d}\right)} \leq N\left(\|f\|_{L_{p}}+\|g\|_{W_{p}^{l / 2, l}\left(\mathbb{R} \times \mathbb{R}^{d-1}\right)}\right),
$$

where $N$ depends only on $d, p$, and $\kappa$. Therefore, to prove (2.16) we need only prove

$$
\|g\|_{W_{p}^{l / 2, l}\left(\mathbb{R} \times \mathbb{R}^{d-1}\right)} \leq N\|f\|_{L_{p}}
$$

where $N$ depends only on $d, p$, and $\kappa$. We prove this inequality as follows. 
Observe that (in the trace sense)

$$
u_{x_{1}}\left(t, 0, x^{\prime}\right)=v_{x_{1}}^{+}\left(t, 0, x^{\prime}\right)+w_{x_{1}}^{+}\left(t, 0, x^{\prime}\right)=v_{x_{1}}^{-}\left(t, 0, x^{\prime}\right)+w_{x_{1}}^{-}\left(t, 0, x^{\prime}\right) .
$$

Thus

$$
v_{x_{1}}^{+}\left(t, 0, x^{\prime}\right)-v_{x_{1}}^{-}\left(t, 0, x^{\prime}\right)=w_{x_{1}}^{-}\left(t, 0, x^{\prime}\right)-w_{x_{1}}^{+}\left(t, 0, x^{\prime}\right) .
$$

Set

$$
h\left(t, x^{\prime}\right)=w_{x_{1}}^{-}\left(t, 0, x^{\prime}\right)-w_{x_{1}}^{+}\left(t, 0, x^{\prime}\right) .
$$

Then by Theorem 2.4 we have $h \in W_{p}^{(l-1) / 2, l-1}\left(\mathbb{R} \times \mathbb{R}^{d-1}\right)$. In addition, from the inequality (2.2) and the estimates for $w^{ \pm}$in (2.17) and (2.18), we have

$$
\|h\|_{W_{p}^{(l-1) / 2, l-1}\left(\mathbb{R} \times \mathbb{R}^{d-1}\right)} \leq N\|f\|_{L_{p}},
$$

where $N$ depends only on $d, p$, and $\kappa$. From this inequality, we notice that the inequality (2.19) follows if we prove

$$
\|g\|_{W_{p}^{l / 2, l}\left(\mathbb{R} \times \mathbb{R}^{d-1}\right)} \leq N\|h\|_{W_{p}^{(l-1) / 2, l-1}\left(\mathbb{R} \times \mathbb{R}^{d-1}\right)} .
$$

Notice that by Lemma 2.8, (2.20), and the definition of $z\left(s, \xi^{\prime}\right)$,

$$
z\left(s, \xi^{\prime}\right) \widetilde{g}\left(s, \xi^{\prime}\right)=\widetilde{h}\left(s, \xi^{\prime}\right), \quad \text { i.e., } \quad \tilde{g}\left(s, \xi^{\prime}\right)=\frac{1}{z\left(s, \xi^{\prime}\right)} \tilde{h}\left(s, \xi^{\prime}\right) .
$$

Then Lemma 2.7 proves the inequality (2.21). The theorem is proved.

Now we solve the first case. Specifically, for a given $f \in L_{p}=L_{p}\left(\mathbb{R}^{d+1}\right)$ and $\lambda>0$, there exists a unique $u \in W_{p}^{1,2}\left(\mathbb{R} \times \mathbb{R}^{d}\right)$ such that

$$
u_{t}=L_{0} u-\lambda u+f
$$

This can be done, as is well known, using the estimate in Theorem 2.3 and the method of continuity. In addition, we can find a unique solution $u \in W_{p}^{1,2}((0, T) \times$ $\mathbb{R}^{d}$ ) of the equation

$$
\left\{\begin{array}{l}
u_{t}=L_{0} u-\lambda u+f \quad \text { in } \quad(0, T) \times \mathbb{R}^{d} \\
u(0, x)=0
\end{array},\right.
$$

where $0<T \leq \infty$.

We now consider the general case. That is, we deal with

$$
u_{t}(t, x)=L u(t, x)-\lambda u(t, x)+f(t, x) \text { in } \mathbb{R} \times \mathbb{R}^{d},
$$

where the coefficients of $L$ satisfy Assumption 2.1 and 2.2. The problem can be solved by freezing the coefficients $a_{j k}$ and using the estimate in Theorem 2.3. Indeed, we can proceed as follows. 
Lemma 2.9. There exist constants $\varepsilon>0, \lambda_{0}$, and $N$, depending only on $d, K, p$, $\kappa$, and $\omega$, such that if $u \in C_{0}^{\infty}\left(\mathbb{R}^{d+1}\right)$ satisfies

$$
\operatorname{supp} u \subset\left\{\left(t, x_{1}, x^{\prime}\right),:\left|t-t_{0}\right|<\varepsilon,\left|x^{\prime}-x_{0}^{\prime}\right|<\varepsilon\right\}
$$

for some $\left(t_{0}, x_{0}^{\prime}\right) \in \mathbb{R} \times \mathbb{R}^{d-1}$, then

$$
\lambda\|u\|_{L_{p}}+\left\|u_{t}\right\|_{L_{p}}+\left\|u_{x x}\right\|_{L_{p}} \leq N\left\|L u-\lambda u-u_{t}\right\|_{L_{p}}
$$

for any $\lambda>\lambda_{0}$.

Proof. We can assume that $t_{0}=0$ and $x_{0}^{\prime}=0$. Choose an infinitely differentiable function $\eta\left(x_{1}\right)$ defined on $\mathbb{R}$ satisfying $\eta \geq 0$ and

$$
\eta\left(x_{1}\right)=\left\{\begin{array}{l}
1 \quad-\varepsilon / 2 \leq x_{1} \leq \varepsilon / 2 \\
0 \quad x_{1} \geq \varepsilon \text { or } x_{1} \leq-\varepsilon
\end{array},\right.
$$

where $\varepsilon$ will be specified later. Set $\mu=1-\eta$.

First notice that $\mu u \in W_{p}^{1,2}\left(\mathbb{R} \times \mathbb{R}_{+}^{d}\right), \mu u \in W_{p}^{1,2}\left(\mathbb{R} \times \mathbb{R}_{-}^{d}\right)$, and $(\mu u)\left(t, 0, x^{\prime}\right)=0$. Thus by the well-known result (see, e.g., Chapter VII and Exercise 7.5 in [9]), there exist $\lambda^{\prime}$ and $N$, depending only on $d, K, p, \kappa$, and $\omega$, such that, for any $\lambda>\lambda^{\prime}$,

$$
\begin{aligned}
& \lambda\|\mu u\|_{L_{p}\left(\mathbb{R} \times \mathbb{R}_{+}^{d}\right)}+\left\|(\mu u)_{t}\right\|_{L_{p}\left(\mathbb{R} \times \mathbb{R}_{+}^{d}\right)}+\left\|(\mu u)_{x x}\right\|_{L_{p}\left(\mathbb{R} \times \mathbb{R}_{+}^{d}\right)} \\
& \leq N\left\|L(\mu u)-\lambda(\mu u)-(\mu u)_{t}\right\|_{L_{p}\left(\mathbb{R} \times \mathbb{R}_{+}^{d}\right)} .
\end{aligned}
$$

The same estimate holds true with $\mathbb{R}_{-}^{d}$ in place of $\mathbb{R}_{+}^{d}$. Hence we have

$$
\lambda\|\mu u\|_{L_{p}}+\left\|(\mu u)_{t}\right\|_{L_{p}}+\left\|(\mu u)_{x x}\right\|_{L_{p}} \leq N\left\|L(\mu u)-\lambda(\mu u)-(\mu u)_{t}\right\|_{L_{p}}
$$

for any $\lambda>\lambda^{\prime}$. Note that the above $N$ is independent of $\varepsilon$.

Now consider $\eta u$. We see that

$$
\operatorname{supp}(\eta u) \subset\left\{\left(t, x_{1}, x^{\prime}\right):|t| \leq \varepsilon,\left|x_{1}\right| \leq \varepsilon,\left|x^{\prime}\right| \leq \varepsilon\right\} .
$$

Set

$$
L_{0}=\left\{\begin{array}{ccc}
a_{j k}^{+}(0) D_{j k} & \text { if } & x_{1}>0 \\
a_{j k}^{-}(0) D_{j k} & \text { if } & x_{1}<0,
\end{array}\right.
$$

where $0 \in \mathbb{R}^{d+1}$. Then by Theorem 2.3 , we have

$$
\lambda\|\eta u\|_{L_{p}}+\left\|(\eta u)_{t}\right\|_{L_{p}}+\left\|(\eta u)_{x x}\right\|_{L_{p}} \leq N\left\|L_{0}(\eta u)-\lambda(\eta u)-(\eta u)_{t}\right\|_{L_{p}}
$$

for $\lambda>0$, where $N$ is the same as in Theorem 2.3. Observe that

$$
\begin{aligned}
& \left\|L_{0}(\eta u)-\lambda(\eta u)-(\eta u)_{t}\right\|_{L_{p}} \\
& \quad \leq\left\|L(\eta u)-\lambda(\eta u)-(\eta u)_{t}\right\|_{L_{p}}+\left\|\left(L_{0}-L\right)(\eta u)\right\|_{L_{p}}
\end{aligned}
$$


and

$$
\left\|\left(L_{0}-L\right)(\eta u)\right\|_{L_{p}} \leq N\left\|(\eta u)_{x}\right\|_{L_{p}}+N\|\eta u\|_{L_{p}}+J_{1}+J_{2},
$$

where $N$ depends only on $d$ and $K$, and

$$
\begin{aligned}
& J_{1}=\sup _{\substack{(t, x) \in \operatorname{supp}(\eta u) \\
x_{1} \geq 0}}\left(\left|a_{j k}^{+}(0)-a_{j k}^{+}(t, x)\right|\right)\left\|(\eta u)_{x x}\right\|_{L_{p}\left(\mathbb{R} \times \mathbb{R}_{+}^{d}\right)},
\end{aligned}
$$

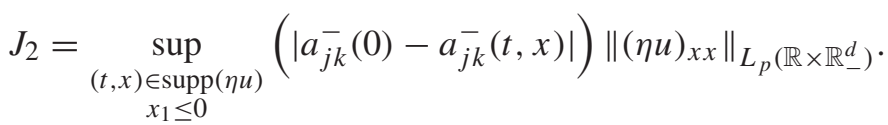

Also observe that

$$
\begin{aligned}
\left|a_{j k}^{+}(0)-a_{j k}^{+}\left(t, x_{1}, x^{\prime}\right)\right| & \leq\left|a_{j k}^{+}(0)-a_{j k}^{+}\left(t, 0, x^{\prime}\right)\right|+\left|a_{j k}^{+}\left(t, 0, x^{\prime}\right)-a_{j k}^{+}\left(t, x_{1}, x^{\prime}\right)\right| \\
& \leq \omega\left(|t|+\left|x^{\prime}\right|\right)+\omega\left(\left|x_{1}\right|\right) .
\end{aligned}
$$

Similarly,

$$
\left|a_{j k}^{-}(0)-a_{j k}^{-}\left(t, x_{1}, x\right)\right| \leq \omega\left(|t|+\left|x^{\prime}\right|\right)+\omega\left(\left|x_{1}\right|\right) .
$$

Hence we can find a sufficiently small $\varepsilon>0$ depending only on $d, K, p, \kappa$, and $\omega$, such that the following inequality holds.

$$
\begin{aligned}
& \lambda\|\eta u\|_{L_{p}}+\left\|(\eta u)_{t}\right\|_{L_{p}}+\left\|(\eta u)_{x x}\right\|_{L_{p}} \\
\leq & N\left\|L(\eta u)-\lambda(\eta u)-(\eta u)_{t}\right\|_{L_{p}}+\frac{1}{4}\left\|(\eta u)_{x x}\right\|_{L_{p}}+N\left\|(\eta u)_{x}\right\|_{L_{p}}+N\|\eta u\|_{L_{p}} .
\end{aligned}
$$

Using the interpolation inequality, we find that the right hand side of the above inequality is not greater than

$$
N\left\|L(\eta u)-\lambda(\eta u)-(\eta u)_{t}\right\|_{L_{p}}+\frac{1}{2}\left\|(\eta u)_{x x}\right\|_{L_{p}}+N^{\prime}\|\eta u\|_{L_{p}},
$$

where $N$ is the same as in (2.25) and $N^{\prime}$ depends only on $d, K, p$, and $\kappa$. This implies that there exits $\lambda^{\prime \prime}>0$ (specifically, $\lambda^{\prime \prime} \geq 2 N^{\prime}$ ) depending only on $d, K, p$, and $\kappa$, such that

$$
\lambda\|\eta u\|_{L_{p}}+\left\|(\eta u)_{t}\right\|_{L_{p}}+\left\|(\eta u)_{x x}\right\|_{L_{p}} \leq 2 N\left\|L(\eta u)-\lambda(\eta u)-(\eta u)_{t}\right\|_{L_{p}}
$$

for any $\lambda>\lambda^{\prime \prime}$, where $N$ is the one in (2.25). From this inequality and the inequality (2.24) we can find $\lambda_{0}$ and $N$ depending only on $d, K, p, \kappa$, and $\omega$, such that the inequality (2.23) holds true. The lemma is proved.

Lemma 2.10. There exist $\lambda_{1}$ and $N$, depending only on $d, K, p, \kappa$, and $\omega$, such that, for any $\lambda>\lambda_{1}$ and $u \in C_{0}^{\infty}\left(\mathbb{R}^{d+1}\right)$, the estimate (2.23) holds true. 
Proof. First note that (recall again that $L_{p}=L_{p}\left(\mathbb{R}^{d+1}\right)$ )

$$
\begin{aligned}
\left\|u_{t}\right\|_{L_{p}} & =\left\|L u-\lambda u-\left(L u-\lambda u-u_{t}\right)\right\|_{L_{p}} \\
& \leq\left\|L u-\lambda u-u_{t}\right\|_{L_{p}}+N\left\|u_{x x}\right\|_{L_{p}}+N\left\|u_{x}\right\|_{L_{p}}+(\lambda+N)\|u\|_{L_{p}} .
\end{aligned}
$$

Thus we need only to prove that there exist $\lambda_{1}$ and $N$ such that, for $\lambda>\lambda_{1}$,

$$
\lambda\|u\|_{L_{p}}+\left\|u_{x x}\right\|_{L_{p}} \leq N\left\|L u-\lambda u-u_{t}\right\|_{L_{p}} .
$$

Choose an infinitely differentiable function $\zeta\left(t, x^{\prime}\right)$ defined on $\mathbb{R}^{d}$ (recall that $t \in \mathbb{R}$, $x^{\prime} \in \mathbb{R}^{d-1}$ ) such that $\|\zeta\|_{L_{p}\left(\mathbb{R}^{d}\right)}=1$ and

$$
\operatorname{supp} \zeta \subset\left\{\left(t, x^{\prime}\right) \in \mathbb{R} \times \mathbb{R}^{d-1}:|t|<\varepsilon,\left|x^{\prime}\right|<\varepsilon\right\},
$$

where $\varepsilon$ is as in Lemma 2.9. We denote

$$
\zeta^{s, y^{\prime}}\left(t, x^{\prime}\right)=\zeta\left(t-s, x^{\prime}-y^{\prime}\right)
$$

Then

$$
\left|u_{x x}(t, x)\right|^{p}=\int_{\mathbb{R}^{d}}\left|u_{x x}(t, x) \zeta^{s, y^{\prime}}\left(t, x^{\prime}\right)\right|^{p} d s d y^{\prime}
$$

Using the fact that $u_{x_{j} x_{k}} \zeta=(u \zeta)_{x_{j} x_{k}}-u_{x_{j}} \zeta_{x_{k}}-u_{x_{k}} \zeta_{x_{j}}-u \zeta_{x_{j} x_{k}}$, we obtain

$$
\begin{aligned}
\left|u_{x_{j} x_{k}}(t, x) \zeta^{s, y^{\prime}}\left(t, x^{\prime}\right)\right|^{p} \leq 2^{p} \mid & \left.\left(u(t, x) \zeta^{s, y^{\prime}}\left(t, x^{\prime}\right)\right)_{x_{j} x_{k}}\right|^{p} \\
& +N\left(|u(t, x)|^{p}+\left|u_{x}(t, x)\right|^{p}\right) \varphi\left(t-s, x^{\prime}-y^{\prime}\right),
\end{aligned}
$$

where $\varphi=\left|\zeta_{x}\right|^{p}+\left|\zeta_{x x}\right|^{p} \in L_{1}\left(\mathbb{R}^{d}\right)$. Combining this and (2.26), we have

$$
\left\|u_{x x}\right\|_{L_{p}}^{p} \leq N \int_{\mathbb{R}^{d}}\left\|\left(u \zeta^{s, y^{\prime}}\right)_{x x}\right\|_{L_{p}}^{p} d s d y^{\prime}+N\left(\|u\|_{L_{p}}^{p}+\left\|u_{x}\right\|_{L_{p}}^{p}\right)
$$

For each $\left(s, y^{\prime}\right) \in \mathbb{R}^{d}$, we see that $u(t, x) \zeta^{s, y^{\prime}}\left(t, x^{\prime}\right)$ satisfies the assumptions in Lemma 2.9. Thus

$$
\left\|\left(u \zeta^{s, y^{\prime}}\right)_{x x}\right\|_{L_{p}} \leq N\left\|\left(L-\lambda-\frac{\partial}{\partial t}\right)\left(u \zeta^{s, y^{\prime}}\right)\right\|_{L_{p}}
$$

for $\lambda>\lambda_{0}$, where $\lambda_{0}$ and $N$ are as in Lemma 2.9. We then have

$$
\left\|u_{x x}\right\|_{L_{p}}^{p} \leq N \int_{\mathbb{R}^{d}}\left\|\left(L-\lambda-\frac{\partial}{\partial t}\right)\left(u \zeta^{s, y^{\prime}}\right)\right\|_{L_{p}}^{p} d s d y^{\prime}+N\left(\|u\|_{L_{p}}^{p}+\left\|u_{x}\right\|_{L_{p}}^{p}\right)
$$


Note that

$$
\begin{aligned}
\left(L-\lambda-\frac{\partial}{\partial t}\right)\left(u \zeta^{s, y^{\prime}}\right)= & \zeta^{s, y^{\prime}}\left(L u-\lambda u-u_{t}\right) \\
& +2 a_{j k} u_{x_{j}} \zeta_{x_{k}}^{s, y^{\prime}}+u\left[a_{j k} \zeta_{x_{j} x_{k}}^{s, y^{\prime}}+b_{j} \zeta_{x_{j}}^{s, y^{\prime}}-\zeta_{t}^{s, y^{\prime}}\right],
\end{aligned}
$$

where, for instance, $\zeta_{x_{j} x_{k}}^{s, y^{\prime}}\left(t, x^{\prime}\right)=\zeta_{x_{j} x_{k}}\left(t-s, x^{\prime}-y^{\prime}\right)$. Thus

$$
\begin{aligned}
\int_{\mathbb{R}^{d}} \|\left(L-\lambda-\frac{\partial}{\partial t}\right)\left(u \zeta^{s, y^{\prime}}\right) & \|_{L_{p}}^{p} d s d y^{\prime} \\
& \leq N\left(\left\|L u-\lambda u-u_{t}\right\|_{L_{p}}^{p}+\|u\|_{L_{p}}^{p}+\left\|u_{x}\right\|_{L_{p}}^{p}\right) .
\end{aligned}
$$

From this and (2.27) we have

$$
\left\|u_{x x}\right\|_{L_{p}}^{p} \leq N\left(\left\|L u-\lambda u-u_{t}\right\|_{L_{p}}^{p}+\|u\|_{L_{p}}^{p}+\left\|u_{x}\right\|_{L_{p}}^{p}\right) .
$$

Similarly,

$$
\begin{aligned}
\lambda^{p}\|u\|_{L_{p}}^{p}=\lambda^{p} \int_{\mathbb{R}^{d}} \| u \zeta^{s, y^{\prime}} & \|_{L_{p}}^{p} d s d y^{\prime} \\
& \leq N\left(\left\|L u-\lambda u-u_{t}\right\|_{L_{p}}^{p}+\|u\|_{L_{p}}^{p}+\left\|u_{x}\right\|_{L_{p}}^{p}\right) .
\end{aligned}
$$

Therefore, from (2.28) and (2.29) we have

$$
\lambda\|u\|_{L_{p}}+\left\|u_{x x}\right\|_{L_{p}} \leq N_{1}\left\|L u-\lambda u-u_{t}\right\|_{L_{p}}+N_{2}\left(\left\|u_{x}\right\|_{L_{p}}+\|u\|_{L_{p}}\right),
$$

where $N_{1}$ and $N_{2}$ depend only on $d, K, p, \kappa$, and $\omega$. Now we make use of the argument in the proof of Lemma 2.9 to finish the proof.

Using the estimate proved in the above lemma and the method of continuity, we arrive at the following theorem.

Theorem 2.11. Let $\lambda>\lambda_{1}$, where $\lambda_{1}$ is as in Lemma 2.10. For $f \in L_{p}\left(\mathbb{R} \times \mathbb{R}^{d}\right)$, there exists a unique solution $u \in W_{p}^{1,2}\left(\mathbb{R} \times \mathbb{R}^{d}\right)$ satisfying the parabolic equation (2.22). Moreover, the solution u satisfies the estimate (2.23).

Remark 2.12. It follows from Theorem 2.11 that there exists a unique solution $u \in W_{p}^{1,2}\left((0, T) \times \mathbb{R}^{d}\right)$ to the equation

$$
\left\{\begin{array}{l}
u_{t}(t, x)=L u(t, x)+f(t, x) \quad \text { in } \quad(0, T) \times \mathbb{R}^{d} . \\
u(0, x)=0
\end{array}\right.
$$

Moreover, the solution $u$ satisfies

$$
\|u\|_{W_{p}^{1,2}\left((0, T) \times \mathbb{R}^{d}\right)} \leq N\|f\|_{L_{p}\left((0, T) \times \mathbb{R}^{d}\right)},
$$

where $N$ depends on $d, K, T, p, \kappa$, and $\omega$. 


\section{Uniqueness of diffusions with discontinuous coefficients}

In this section we investigate the well-posedness of the martingale problem for $L_{t}$

$$
L_{t}=\frac{1}{2} a_{j k}(t, \cdot) \frac{\partial^{2}}{\partial x_{j} \partial x_{k}}+b_{j}(t, \cdot) \frac{\partial}{\partial x_{j}},
$$

where the coefficient $a_{j k}$ and $b_{j}$ satisfy the following assumptions.

Assumption 3.1. $a_{j k}(t, x)$ and $b_{j}(t, x)$ are Borel measurable functions defined on $[0, \infty) \times \mathbb{R}^{d}$ such that, for all $t \in[0, \infty), x, \vartheta \in \mathbb{R}^{d}$,

$$
\begin{aligned}
& a_{j k}=a_{k j}, \quad a_{j k}(t, x) \vartheta_{j} \vartheta_{k} \geq \kappa|\vartheta|^{2}, \\
& \left|a_{j k}(t, x)\right|+\left|b_{j}(t, x)\right|^{2} \leq K\left(1+|x|^{2}\right) .
\end{aligned}
$$

Assumption 3.2. Let $I$ be the set of all integers. There exists a sequence $\left\{\gamma_{j}, j \in\right.$ I\} $\subset \mathbb{R}, \gamma_{j}<\gamma_{j+1}$, with no limit points such that

(i) for $x=\left(x_{1}, x^{\prime}\right), x_{1} \notin\left\{\gamma_{j}, j \in I\right\}$,

$$
\lim _{y \rightarrow x} \sup _{0 \leq t \leq T}\left|a_{j k}(t, y)-a_{j k}(t, x)\right|=0
$$

for all $T>0$,

(ii) for $x=\left(\gamma_{j}, x^{\prime}\right), j \in I$, there exist $a_{j k}^{+}\left(t, \gamma_{j}, x^{\prime}\right)$ and $\bar{a}_{j k}^{-}\left(t, \gamma_{j}, x^{\prime}\right)$ defined on $[0, \infty) \times \mathbb{R}^{d-1}$ such that they are continuous (in $\left(t, x^{\prime}\right)$ ), and they satisfy

$$
\lim _{\substack{\left(y_{1}, y^{\prime}\right) \rightarrow\left(\gamma_{j}, x^{\prime}\right) \\ y_{1}>\gamma_{j}}} \sup _{\substack{0 \leq t \leq T \\ \gamma_{j k}}}\left|a_{j k}^{+}\left(t, \gamma_{j}, x^{\prime}\right)-a_{j k}\left(t, y_{1}, y^{\prime}\right)\right|=0
$$

and

$$
\lim _{\substack{\left(y_{1}, y^{\prime}\right) \rightarrow\left(\gamma_{j}, x^{\prime}\right) \\ y_{1}<\gamma_{j}}} \sup _{\substack{0 \leq t \leq T \\ 0}}\left|a_{j k}^{-}\left(t, \gamma_{j}, x^{\prime}\right)-a_{j k}\left(t, y_{1}, y^{\prime}\right)\right|=0
$$

for all $T>0$.

Let $\Omega=C\left([0, \infty), \mathbb{R}^{d}\right)$ and $\mathcal{F}=\mathcal{B}(\Omega)$, where $C\left([0, \infty), \mathbb{R}^{d}\right)$ is the Polish space of continuous $\mathbb{R}^{d}$-valued functions given on $[0, \infty)$ with metric

$$
d\left(\omega_{1}, \omega_{2}\right)=\sum_{n=1}^{\infty} \frac{1}{2^{n}} \max _{0 \leq t \leq n}\left(\left|\omega_{1}(t)-\omega_{2}(t)\right| \wedge 1\right) .
$$

$\mathcal{B}(\Omega)$ is, as usual, the Borel $\sigma$-field of subsets of $\Omega$. Define a random variable $X$ on $\Omega$ by $X_{t}(\omega)=\omega(t), \omega \in \Omega$. 
We say that the martingale problem for $L_{t}$ (or martingale problem for $a_{j k}$ and $b_{j}$ ) is well-posed (see [15], [4]) if, for each $(s, x) \in[0, \infty) \times \mathbb{R}^{d}$, there exists a unique probability measure $P_{s, x}$ on $(\Omega, \mathcal{F})$ satisfying

$$
P_{s, x}\left(X_{t}=x, \quad 0 \leq t \leq s\right)=1
$$

such that

$$
f\left(X_{t}\right)-\int_{s}^{t} L_{r} f\left(X_{r}\right) d r
$$

is a $P_{s, x}$ - martingale after time $s$ for all $f \in C_{0}^{\infty}\left(\mathbb{R}^{d}\right)$.

Remark 3.3. The well-posedness of the martingale problem for the operator $L_{t}$ in (3.1) is equivalent to that of the stochastic integral equation

$$
\xi_{t}=x+\int_{0}^{t} \sigma\left(r, \xi_{r}\right) d W_{r}+\int_{0}^{t} b\left(r, \xi_{r}\right) d r
$$

where $W_{t}$ is a Brownian motion, $\sigma$ is the positive square root of the matrix $\left[a_{j k}\right]_{j, k=1}^{d}$, and $b=\left(b_{j}\right)_{j=1}^{d}$. The stochastic integral equation is said to be well-posed if, for every initial condition $x \in \mathbb{R}^{d}$, it admits a weak solution which is unique in the sense of probability law. For details, see [4].

We first note that, for each $(s, x) \in[0, \infty) \times \mathbb{R}^{d}$, there exists a solution to the martingale problem for $L_{t}$. This follows from Remark 2.1 in [7] and references therein.

To prove uniqueness, we start with coefficients $a_{j k}$ and $b_{j}$ which satisfy assumptions in Section 2.

Lemma 3.4. Let $a_{j k}(t, x)$ and $b_{j}(t, x)$ be Borel measurable functions defined on $[0, \infty) \times \mathbb{R}^{d}$ satisfying Assumption 2.1 and 2.2 in Section 2. Then the martingale problem for $L_{t}$ is well-posed.

Proof. Clearly, we need to prove only the uniqueness of a solution to martingale problem for $a_{j k}$ and $b_{j}$ starting from $(s, x) \in[0, \infty) \times \mathbb{R}^{d}$. By Corollary 6.2.6 in [15], this can be done if we prove that, for each $0 \leq s<T$ and $f \in C_{0}^{\infty}([s, T] \times$ $\mathbb{R}^{d}$ ), there is a bounded measurable function $u$ defined on $[0, T) \times \mathbb{R}^{d}$ such that

$$
u(s, x)=-E^{P}\left[\int_{s}^{T} f\left(t, X_{t}\right) d t\right]
$$

whenever $P$ solves the martingale problem for $L_{t}$ starting from $(s, x) \in[0, \infty) \times$ $\mathbb{R}^{d}$.

Let $P=P_{s, x}$ is a solution to the martingale problem for $L_{t}$ starting from $(s, x) \in[0, \infty) \times \mathbb{R}^{d}$. For a given $f \in C_{0}^{\infty}\left([s, T] \times \mathbb{R}^{d}\right)$, we set

$$
\tilde{f}=\left\{\begin{aligned}
f(t, x) & \text { if } \quad s \leq t \leq T \\
0 & \text { otherwise }
\end{aligned}\right.
$$


We also fix a real number $p$ such that $d+1<p<\infty$. Then by remark 2.12 we can find a unique solution $u \in W_{p}^{1,2}\left((0, T) \times \mathbb{R}^{d}\right)$ of the equation

$$
\left\{\begin{aligned}
u_{t}+L_{t} u(t, x) & =\tilde{f} \quad \text { in } \quad(0, T) \times \mathbb{R}^{d} . \\
u(T, x) & =0
\end{aligned}\right.
$$

Now we find a sequence $u_{n} \in C^{1,2}\left([0, T] \times \mathbb{R}^{d}\right)$ such that each $u_{n}$ is bounded (derivatives are bounded as well), $u_{n} \rightarrow u$ in $W_{p}^{1,2}\left((0, T) \times \mathbb{R}^{d}\right)$, and $u_{n}(T, x)=0$. Note that we have (see Lemma 6.3.1 in [15])

$$
u_{n}(s, x)=-E^{P}\left[\int_{s}^{T}\left(u_{n t}+L_{t} u_{n}\right)\left(t, X_{t}\right) d t\right]
$$

for each $n$, where, as we recall,

$$
\begin{aligned}
\left(u_{n t}+L u_{n}\right) & \left(t, X_{t}\right) \\
& =u_{n t}\left(t, X_{t}\right)+\frac{1}{2} a_{j k}\left(t, X_{t}\right) u_{n x_{j} x_{k}}\left(t, X_{t}\right)+b_{j}\left(t, X_{t}\right) u_{n x_{j}}\left(t, X_{t}\right) .
\end{aligned}
$$

Since $u_{n}(s, x) \rightarrow u(s, x)$ pointwise by the Sobolev embedding theorem, to prove (3.2), it only remains to prove that

$$
E^{P}\left[\int_{s}^{T}\left(u_{n t}+L_{t} u_{n}\right)\left(t, X_{t}\right) d t\right] \rightarrow E^{P}\left[\int_{s}^{T}\left(u_{t}+L_{t} u\right)\left(t, X_{t}\right) d t\right] .
$$

This follows easily from

$$
\begin{gathered}
E^{P} \int_{s}^{T}\left|\left(u_{t}^{n}+L_{t} u^{n}\right)\left(t, X_{t}\right)-\left(u_{t}+L_{t} u\right)\left(t, X_{t}\right)\right| d t \\
\quad \leq N\left\|\left(u_{n t}+L_{t} u_{n}\right)-\left(u_{t}+L_{t} u\right)\right\|_{L_{p}\left((0, T) \times \mathbb{R}^{d}\right)} \\
\quad \leq N\left\|u_{n}-u\right\|_{W_{p}^{1,2}\left((0, T) \times \mathbb{R}^{d}\right)} \rightarrow 0,
\end{gathered}
$$

where the first inequality is due to Theorem 2.3.4 in [6]. The lemma is proved.

Now we can say that the martingale problem for $L_{t}$, where coefficients $a_{j k}$ and $b_{j}$ satisfying Assumption 3.1 and 3.1, is well-posed. The justification of this claim follows from Corollary 10.1.2 and Theorem 6.6.1 in [15]. Indeed, we may prove this as follows.

As noted earlier, we have the existence for the martingale problem for $L_{t}$. Thus by Corollary 10.1.2 in [15] we need only check the well-posedness of the martingale problem for $L_{t}$ with bounded $a_{j k}$ and $b_{j}$ satisfying Assumption 3.1 and 3.2. In that case by Theorem 6.6.1 (and Exercise 6.7.4) in [15] it is enough to show that, for each $(s, x) \in[0, \infty) \times \mathbb{R}^{d}$, there exist coefficients $\tilde{a}_{j k}$ and $\tilde{b}_{j}$ defined on $[0, \infty) \times \mathbb{R}^{d}$ 
such that

(i) $\tilde{a}_{j k}=a_{j k}$ and $\tilde{b}_{j}=b_{j}$ in a neighborhood of $(s, x)$,

(ii) the martingale for $\tilde{L}_{t}$ is well-posed, where

$$
\tilde{L}_{t}=\frac{1}{2} \tilde{a}_{j k}(t, \cdot) \frac{\partial^{2}}{\partial x_{j} \partial x_{k}}+\tilde{b}_{j}(t, \cdot) \frac{\partial}{\partial x_{j}} .
$$

Note that $\left\{\gamma_{j}, j \in I\right\}$ has no limit points. Thus, for points $(s, x) \in[0, \infty) \times \mathbb{R}^{d}$, where $x_{1} \notin\left\{\gamma_{j}, j \in I\right\}$, we can find coefficients $\tilde{a}_{j k}$ and $\tilde{b}_{j}$ satisfying the above two conditions. Especially, (ii) is satisfied by results in Ch. 7 in [15].

For points $(s, x) \in[0, \infty) \times \mathbb{R}^{d}$, where $x_{1} \in\left\{\gamma_{j}, j \in I\right\}$ (say, for example, $x_{1}=0$ and $\left.0 \in\left\{\gamma_{j}, j \in I\right\}\right)$, we find coefficients $\tilde{a}_{j k}$ and $\tilde{b}_{j}$ defined on $[0, \infty) \times \mathbb{R}^{d}$ such that they satisfy Assumption 2.1 and 2.2 in Section 2 as well as (i) in the above. In fact, we may set

$$
\tilde{a}_{j k}=\varphi a_{j k}+(1-\varphi) \delta_{j k}, \quad \tilde{b}_{j}=b_{j},
$$

where $\varphi$ is a non-negative infinitely differentiable function defined on $\mathbb{R}^{d+1}$ such that $\varphi=1$ in a sufficiently small neighborhood of $\left(s, 0, x^{\prime}\right)$ and $\operatorname{supp} \varphi$ dose not intersect $\left\{\left(t, \gamma_{k}, x^{\prime}\right):\left(t, x^{\prime}\right) \in[0, \infty) \times \mathbb{R}^{d-1}\right\}$ for all $\gamma_{k} \in\left\{\gamma_{j}, j \in I\right\} \backslash\{0\}$. Now we prove (ii), i.e., the well-posedness of the martingale problem for $\tilde{a}_{j k}$ and $\tilde{b}_{j}$, using Lemma 3.4. Therefore, we have proved

Theorem 3.5. Under Assumption 3.1 and 3.2, the martingale problem for $L_{t}$, where $L_{t}$ is as in (3.1), is well-posed.

\section{References}

[1] H. Amann, Compact embeddings of vector-valued Sobolev and Besov spaces, Glas. Mat. 35 (2000), 161-177. Dedicated to the memory of Branko Najman.

[2] R. F. BASS and É. PARDOUX, Uniqueness for diffusions with piecewise constant coefficients, Probab. Theory Related Fields 76(1987), 557-572.

[3] M. C. Cerutti, L. EscauriazA and E. B. Fabes, Uniqueness for some diffusions with discontinuous coefficients, Ann. Probab. 19 (1991), 525-537.

[4] I. Karatzas and S. E. Shreve, "Brownian Motion and Stochastic Calculus", Graduate Texts in Mathematics, Vol. 113, Springer-Verlag, New York, second edition, 1991.

[5] D. KIM, Second order elliptic equations in $\mathbb{R}^{d}$ with piecewise continuous coefficients, Potential Anal., 2005, submitted.

[6] N. V. KRYLOV, "Controlled Diffusion Processes", Applications of Mathematics, Vol. 14, Springer-Verlag, New York, 1980. Translated from the Russian by A. B. Aries.

[7] N. V. KRYLOV, On weak uniqueness for some diffusions with discontinuous coefficients, Stochastic Process. Appl. 113 (2004), 37-64.

[8] O. A. LadyŽEnskaja, V. A. Solonnikov and N. N. URAL'CEVA, "Linear and Quasilinear Equations of Parabolic Type". Translated from the Russian by S. Smith. Translations of Mathematical Monographs, Vol. 23, American Mathematical Society, Providence, R.I., 1967. 
[9] G. M. Lieberman, "Second Order Parabolic Differential Equations", World Scientific Publishing Co. Inc., River Edge, NJ, 1996.

[10] A. LorenZI, On elliptic equations with piecewise constant coefficients, Appl. Anal. 2 (1972), 79-96.

[11] A. LorenZI, On elliptic equations with piecewise constant coefficients, II, Ann. Scuola Norm. Sup. Pisa Cl. Sci. (3) 26 (1972), 839-870.

[12] S. SALSA, Un problema di Cauchy per un operatore parabolico con coefficienti costanti a tratti, Matematiche 31 (1977), 126-146.

[13] L. G. SofTova, Quasilinear parabolic operators with discontinuous ingredients, Nonlinear Anal. 52 (2003), 1079-1093.

[14] E. M. STEIN, "Singular Integrals and Differentiability Properties of Functions", Princeton Mathematical Series, Vol. 30, Princeton University Press, Princeton, N.J., 1970.

[15] D. W. Stroock and S. R. SRinivasa VAradhan, "Multidimensional Diffusion Processes", Grundlehren der Mathematischen Wissenschaften [Fundamental Principles of Mathematical Sciences], Vol. 233, Springer-Verlag, Berlin, 1979.

[16] H. Triebel, "Interpolation Theory, Function Spaces, Differential Operators", NorthHolland Mathematical Library, Vol. 18, North-Holland Publishing Co., Amsterdam, 1978.

[17] P. WeIDEMAIER, Maximal regularity for parabolic equations with inhomogeneous boundary conditions in Sobolev spaces with mixed $L_{p}$-norm, Electron. Res. Announc. Amer. Math. Soc. (electronic), 8 (2002), 47-51.

School of Mathematics

University of Minnesota

127 Vincent Hall

206 Church St. SE

Minneapolis, MN 55455, U.S.A.

dykim@math.umn.edu 